\title{
Optimized MIMO Transmission and Compression for Interference Mitigation with Cooperative Relay
}

\author{
Seyed Arvin Ayoughi and Wei Yu \\ Department of Electrical and Computer Engineering \\ University of Toronto, Toronto, ON, M5S 3G4, Canada \\ Emails: sa.ayoughi@mail.utoronto.ca,weiyu@comm.utoronto.ca
}

\begin{abstract}
This paper considers a novel use of device-to-device link for cooperative communication wherein a nearby user terminal acts as a relay in enabling both signal enhancement and common interference rejection at the intended destination. Assuming Gaussian transmission and Gaussian compress-and-forward relaying strategy for the multiple-input multiple-output (MIMO) relay channel with a finite-capacity out-of-band relay-destination link and with arbitrarily correlated noises, this paper proposes a coordinate ascent approach for iteratively optimizing the transmit covariance matrix at the source and the quantization noise covariance matrix at the relay. We show that the optimization of quantization noise covariance matrix under fixed input can be solved in closed form using a simultaneous diagonalization approach, while the optimization of transmit covariance matrix under fixed quantization can be cast as a convex optimization problem. This paper further introduces the concept of antenna pooling and illustrates the importance of accounting for the noise correlation across the user terminals due to common interference. We show that the optimized transmission and device-to-device relaying strategies that take advantage of the noise correlation can significantly improve the user throughput in a cellular environment by enabling interference rejection across the user terminals.
\end{abstract}

\section{INTRODUCTION}

Provisioning of high-rate downlink transmission for cell-edge users is a central challenge in the design of wireless cellular networks. A user at the edge of a cell has to combat not only the relatively weak direct signal from its own base-station, but also strong interference from the neighboring base-stations. This paper proposes a novel signal-enhancing and interferencemitigation strategy for the downlink based on the following key observation. Modern user terminals are often capable of establishing high-capacity out-of-band device-to-device links (e.g., by using WiFi or Bluetooth), especially when the terminals are located close to each other. The physical proximity of the user terminals also suggests that the interference at the multiple terminals can be highly correlated. This opens up the possibility of utilizing device-to-device communication for not only signal enhancement but also interference mitigation.

Intuitively, if the device-to-device cooperation link has infinite capacity, the cooperating user terminals can essentially pool their antennas together for joint reception. Multi-antenna joint reception can significantly improve the downlink throughput for cell-edge users because it not only enhances the detection of the intended signal, but also allows the mitigation of common outof-cell interference across the user terminals. The main aim of this paper is to show that antenna pooling is an effective strategy even when the cooperation link capacity is finite. Toward this end, this paper models the device-to-device cooperation scenario as a relay channel with a finite-capacity link from the relay to the destination. To model the common interference, the noises at the relay and the destination are assumed to be arbitrarily correlated. The base-station and the cooperating user terminals are all assumed to be equipped with multiple antennas. The objective of this paper is to jointly optimize the transmission and relaying strategy and to characterize the resulting achievable rate for the multiple-input multiple-output (MIMO) relay channel.

The joint optimization of the MIMO transmission and relaying strategies is a difficult task, because the information theoretical capacity of the relay channel is still an open problem [1], [2]. This paper adopts a compress-and-forward relaying strategy (which is appropriate given the physical proximity of the relay and the destination), further restricts both the source signal and the relay quantization noise to be Gaussian, and focuses on the joint optimization of the transmit covariance and the relay quantization noise covariance matrices. Such an optimization is still quite challenging, because the overall achievable rate is not a concave function of the optimization variables; also the constraint is not convex-in fact both are concave in transmit covariance and convex in quantization noise covariance.

This paper shows that under fixed transmit covariance, the optimization of the quantization noise covariance can be solved in closed form using a simultaneous diagonalization by *congruence approach [3], while the optimization of the input covariance under fixed quantization noise covariance can be cast as a convex logdet problem. Together, the iterative optimization between the two steps gives a stationary point of the Lagrangian, and leads to transmit and quantization noise covariance matrices that can take advantage of the device-to-device cooperation link appropriately. Optimized relaying leads to significant throughput improvement by enabling joint reception and interference rejection across the multi-antenna relay and destination terminals.

The Gaussian single-antenna relay channel with correlated noises has been studied previously [4] [5]. Compress-andforward, which uses Wyner-Ziv coding to take advantage of the side information at the receiver, is shown to be able to exploit the noise correlation. It in fact achieves the capacity of such a channel to within a constant gap in the single-antenna case [5].

For the MIMO relay channel, the joint optimization of transmit and quantization noise covariance matrices is more challenging. Toward this end, [6] suggests an alternating optimization 
approach between the transmit and quantization noise covariance matrices, where each step of the iteration is required to satisfy the relay link capacity constraint with equality. However, the convergence of the algorithm in [6] cannot be guaranteed [7]. This paper resolves this difficulty by proposing an alternating optimization of the Lagrangian.

A key step in the proposed alternating optimization approach involves the optimization of the quantization noise covariance under fixed input. This problem has been considered in [8] for a MIMO relay channel with independent noises, for which the optimal solution is shown to be related to the eigenvalues of certain conditional covariance matrix. This paper focuses on the MIMO relay channel with correlated noises for which the solution is more complex. In this context, [9] draws a connection between the quantization noise covariance matrix optimization problem with certain source coding problem and provides a solution using the canonical correlation analysis technique in statistics. In this paper, we use a different technique of simultaneous diagonalization by *congruence [3] of two conditional covariance matrices, and obtain a closed-form solution to the stationary point of the Lagrangian based on the generalized eigenvalues of the two matrices. Although the solution to the optimization problem can eventually be shown to be the same as that of [9], the diagonalization approach in this paper is simpler and provides insight about the structure of the optimal MIMO relaying strategy.

\section{System Model And Problem Formulation}

Consider a wireless MIMO cellular network setting with a base-station and two cell-edge users as depicted in Fig. 1, where one user terminal cooperates with the other terminal by relaying information through an independent digital link of finite capacity using a different frequency band. We assume common sources of interference from neighboring base-stations, resulting in correlated noises at the relay and the destination.

Mathematically, the communication scenario is modeled as a Gaussian MIMO relay channel with an out-of-band relaydestination link of fixed capacity $C_{0}$ bits per channel use. The source, relay, and destination are equipped with $s, r$, and $d$ antennas, respectively. Let $t$ be the total number of antennas from all the interfering base-stations combined together. The received signals at the relay and destination are modeled as

$$
\begin{gathered}
\mathbf{Y}_{r}=H_{s r} \mathbf{X}+\mathbf{N}_{r}, \\
\mathbf{Y}_{d}=H_{s d} \mathbf{X}+\mathbf{N}_{d},
\end{gathered}
$$

where the Gaussian noises at the relay and the destination are correlated due to a common source of interference, i.e.,

$$
\begin{aligned}
& \mathbf{N}_{r}=H_{t r} \mathbf{X}_{t}+\mathbf{N}_{1}, \\
& \mathbf{N}_{d}=H_{t d} \mathbf{X}_{t}+\mathbf{N}_{2} .
\end{aligned}
$$

Here, $H_{s r} \in \mathbb{C}^{r \times s}$ and $H_{s d} \in \mathbb{C}^{d \times s}$ are the sourcerelay and source-destination channel matrices respectively; $H_{t r} \in \mathbb{C}^{r \times t}$ and $H_{t d} \in \mathbb{C}^{d \times t}$ are the interferers-to-relay and interferers-to-destination channel matrices respectively; $\mathbf{N}_{1} \sim$ $\mathcal{C N}\left(\mathbf{0}_{r \times 1}, \sigma^{2} \mathbf{I}_{r}\right)$ and $\mathbf{N}_{2} \sim \mathcal{C N}\left(\mathbf{0}_{d \times 1}, \sigma^{2} \mathbf{I}_{d}\right)$ are additive and independent background noises at the relay and the destination respectively; $\mathbf{X} \in \mathbb{C}^{s \times 1}$ is the transmit vector from the source under power constraint $P$; finally $\mathbf{X}_{t} \in \mathbb{C}^{t \times 1}$ is the interference signal that is assumed to be Gaussian with $\mathbf{X}_{t} \sim \mathcal{C N}\left(\mathbf{0}_{t \times 1}, S_{\mathbf{X}_{t}}\right)$, independent of everything else, and is treated as a part of noise.

This paper assumes that the relay employs a compress-andforward strategy in which the relay quantizes its observation and sends the quantization index through the digital link to the destination. The quantization process involves Wyner-Ziv coding, which accounts for the fact that the received signal at the destination is correlated with the relay observation (due to both the common source signal and the correlated noises). The achievable rate for compress-and-forward is [1, Theorem 6]:

$$
\begin{array}{cl}
\max _{p(\mathbf{x}) p\left(\hat{\mathbf{y}}_{r} \mid \mathbf{y}_{r}\right)} & I\left(\mathbf{X} ; \widehat{\mathbf{Y}}_{r}, \mathbf{Y}_{d}\right) \\
\text { s.t. } & I\left(\mathbf{Y}_{r} ; \widehat{\mathbf{Y}}_{r} \mid \mathbf{Y}_{d}\right) \leq C_{0} \\
& \mathbb{E}\left\{\mathbf{X}^{\dagger} \mathbf{X}\right\} \leq P .
\end{array}
$$

where $(\cdot)^{\dagger}$ denotes conjugate transpose.

It can be shown that Gaussian quantization at the relay is optimal for Gaussian signaling at the source [9] and vice versa. Thus, this paper restricts attention to Gaussian transmission $\mathbf{X} \sim$ $\mathcal{C N}\left(\mathbf{0}_{s \times 1}, S_{\mathbf{X}}\right)$ and Gaussian quantization modeled as

$$
\widehat{\mathbf{Y}}_{r}=\mathbf{Y}_{r}+\mathbf{Q}
$$

where $\mathbf{Q} \sim \mathcal{C N}\left(\mathbf{0}_{r \times 1}, S_{\mathbf{Q}}\right)$. In this case, the optimization problem becomes

$$
\begin{array}{cl}
\max & f_{o}\left(S_{\mathbf{X}}, S_{\mathbf{Q}}\right) \\
\text { s.t. } & f_{c}\left(S_{\mathbf{X}}, S_{\mathbf{Q}}\right) \leq C_{0} \\
& S_{\mathbf{X}} \succeq \mathbf{0}, S_{\mathbf{Q}} \succeq \mathbf{0}, \operatorname{tr}\left(S_{\mathbf{X}}\right) \leq P,
\end{array}
$$

where the objective function is

$$
\begin{aligned}
& f_{o}\left(S_{\mathbf{X}}, S_{\mathbf{Q}}\right)=I\left(\mathbf{X} ; \widehat{\mathbf{Y}}_{r}, \mathbf{Y}_{d}\right)=h\left(\widehat{\mathbf{Y}}_{r}, \mathbf{Y}_{d}\right)-h\left(\widehat{\mathbf{Y}}_{r}, \mathbf{Y}_{d} \mid \mathbf{X}\right) \\
& =\log \left|H S_{\mathbf{X}} H^{\dagger}+S_{\mathbf{i n t}}+\sigma^{2} \mathbf{I}_{(r+d)}+\left[\begin{array}{cc}
S_{\mathbf{Q}} & \mathbf{0}_{r \times d} \\
\mathbf{0}_{d \times r} & \mathbf{0}_{d \times d}
\end{array}\right]\right| \\
& \quad-\log \left|S_{\mathbf{i n t}}+\sigma^{2} \mathbf{I}_{(r+d)}+\left[\begin{array}{cc}
S_{\mathbf{Q}} & \mathbf{0}_{r \times d} \\
\mathbf{0}_{d \times r} & \mathbf{0}_{d \times d}
\end{array}\right]\right|
\end{aligned}
$$

the constraint is

$$
\begin{aligned}
& f_{c}\left(S_{\mathbf{X}}, S_{\mathbf{Q}}\right)=I\left(\mathbf{Y}_{r} ; \widehat{\mathbf{Y}}_{r} \mid \mathbf{Y}_{d}\right)=h\left(\widehat{\mathbf{Y}}_{r}, \mathbf{Y}_{d}\right)-h\left(\mathbf{Y}_{d}\right)-h(\mathbf{Q}) \\
& =\log \left|H S_{\mathbf{X}} H^{\dagger}+S_{\mathbf{i n t}}+\sigma^{2} \mathbf{I}_{(r+d)}+\left[\begin{array}{cc}
S_{\mathbf{Q}} & \mathbf{0}_{r \times d} \\
\mathbf{0}_{d \times r} & \mathbf{0}_{d \times d}
\end{array}\right]\right| \\
& \quad-\log \left|H_{s d} S_{\mathbf{X}} H_{s d}^{\dagger}+S_{\mathbf{i n t}}^{(2,2)}+\sigma^{2} \mathbf{I}_{d}\right|-\log \left|S_{\mathbf{Q}}\right| ;
\end{aligned}
$$

and $H=\left[H_{s r}^{\dagger} H_{s d}^{\dagger}\right]^{\dagger}$ is the overall channel matrix; finally,

$$
S_{\text {int }}=\left[\begin{array}{ll}
S_{\text {int }}^{(1,1)} & S_{\text {int }}^{(1,2)} \\
S_{\mathbf{i n t}}^{(2,1)} & S_{\mathbf{i n t}}^{(2,2)}
\end{array}\right]=\left[\begin{array}{ll}
H_{t r} S_{\mathbf{X}_{t}} H_{t r}^{\dagger} & H_{t r} S_{\mathbf{X}_{t}} H_{t d}^{\dagger} \\
H_{t d} S_{\mathbf{X}_{t}} H_{t r}^{\dagger} & H_{t d} S_{\mathbf{X}_{t}} H_{t d}^{\dagger}
\end{array}\right]
$$

is the interference covariance matrix. This paper assumes the availability of channel state information and the statistics of noise and interference at a centralized location for solving the optimization problem (7). 


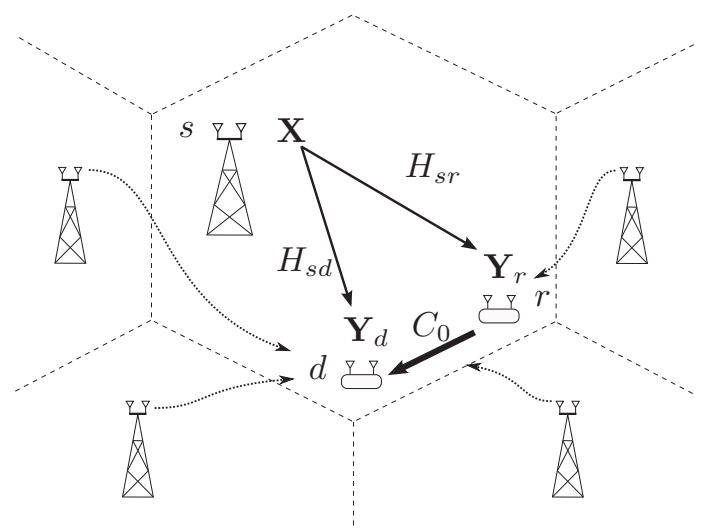

Fig. 1. A wireless cellular network with a base-station and two cell-edge users connected with a finite-capacity device-to-device cooperative relay link. The two users experience correlated noises due to common sources of interference.

\section{OptimizATion Algorithm}

The problem (7) is not a convex optimization problem, as both the objective function and the constraint are concave in $S_{\mathbf{X}}$ and convex in $S_{\mathbf{Q}}$. To tackle this problem, this section first formulates the Lagrangian, then proposes an iterative optimization approach for maximizing the Lagrangian.

\section{A. Iterative Optimization of Lagrangian}

The Lagrangian of the optimization problem (7) is:

$$
\mathcal{L}\left(S_{\mathbf{X}}, S_{\mathbf{Q}}, \mu\right)=f_{o}\left(S_{\mathbf{X}}, S_{\mathbf{Q}}\right)-\mu\left(f_{c}\left(S_{\mathbf{X}}, S_{\mathbf{Q}}\right)-C_{0}\right) .
$$

The overall optimization approach for solving (7) is to find a stationary point of the Lagrangian for a fixed Lagrange multiplier $\mu$, i.e, to solve

$$
\max _{S_{\mathbf{X}} \succeq \mathbf{0}, S_{\mathbf{Q}} \succeq \mathbf{0}, \operatorname{tr}\left(S_{\mathbf{X}}\right) \leq P} \mathcal{L}\left(S_{\mathbf{X}}, S_{\mathbf{Q}}, \mu\right),
$$

then to search for the optimal $\mu^{*}$ that results in

$$
f_{c}\left(S_{\mathbf{X}}^{*}, S_{\mathbf{Q}}^{*}\right)=C_{0}
$$

in an outer loop.

It can be argued that the optimal $\mu^{*}$ must be in $(0,1]$. This is because when $\mu=0$, i.e., when the objective is to maximize the overall rate without relay link capacity constraint, the optimal $S_{\mathbf{Q}}^{*}$ should be a zero matrix, resulting in $f_{c}\left(S_{\mathbf{X}}^{*}, S_{\mathbf{Q}}^{*}\right)=+\infty$. When $\mu \geq 1$, i.e., the relay link rate constraint penalizes the objective at more than a $1: 1$ ratio, the optimal $S_{\mathbf{Q}}^{*}$ should be infinite, resulting in $f_{c}\left(S_{\mathbf{X}}^{*}, S_{\mathbf{Q}}^{*}\right)=0$.

The rest of this paper focuses on solving the optimization problem (12) with a fixed $\mu \in(0,1]$. The outer loop for searching for the optimal $\mu^{*}$ is relatively straightforward, as it is a one-dimensional root-finding problem that can be solved using a standard bisection approach.

This paper proposes an iterative coordinate ascent approach for solving (12). The idea is to find the optimal $S_{\mathrm{Q}}$ that maximizes $\mathcal{L}\left(S_{\mathbf{X}}, S_{\mathbf{Q}}, \mu\right)$ for a fixed $S_{\mathbf{X}}$, then to find the optimal $S_{\mathbf{X}}$ that maximizes $\mathcal{L}\left(S_{\mathbf{X}}, S_{\mathbf{Q}}, \mu\right)$ for a fixed $S_{\mathbf{Q}}$, and to iterate between the two steps. It can be shown that for a fixed $\mu \in(0,1]$, each of the individual optimizations of $S_{\mathbf{X}}$ and $S_{\mathbf{Q}}$

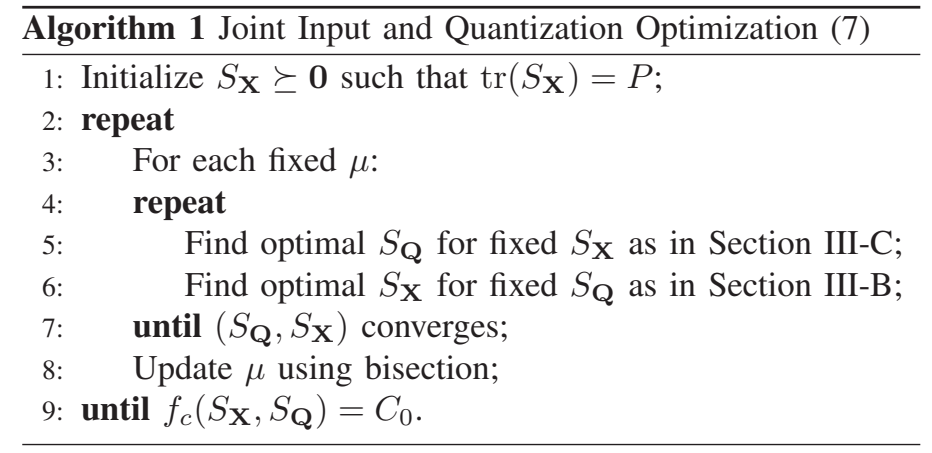

can be solved to global optimality (and the global optimum is essentially unique). The iterative optimization process provides a nondecreasing sequence of the Lagrangian objective, so the iterative process must converge. The convergent point is a stationary point.

The detailed algorithms for optimizing $S_{\mathbf{Q}}$ for a fixed $S_{\mathbf{X}}$ and for optimizing $S_{\mathbf{X}}$ for a fixed $S_{\mathbf{Q}}$ are provided in subsequent sections. The overall iterative approach is summarized as Algorithm 1. The following theorem states the convergence result formally.

Theorem 1 Assuming that the optimal $S_{\mathbf{X}}$ for a fixed $S_{\mathbf{Q}}$ is unique and the optimal $S_{\mathrm{Q}}$ for a fixed $S_{\mathbf{X}}$ is unique, the inner iterative optimization procedure in Algorithm 1 converges to a stationary point of the Lagrangian maximization problem (12). Further, the optimal $\mu$ is one that satisfies (13). Such a $\mu$ leads to a Karush-Kuhn-Tucker (KKT) point of the joint transmit and quantization noise covariance optimization problem (7).

Proof: For a fixed $\mu$, coordinate ascent on the Lagrangian is monotonically increasing, so it converges. The uniqueness in the optimization of $S_{\mathbf{X}}$ for a fixed $S_{\mathbf{Q}}$ and in the optimization of $S_{\mathrm{Q}}$ for a fixed $S_{\mathbf{X}}$ ensures that coordinate ascent converges to a stationary point. This together with a $\mu$ that satisfies (13) gives a KKT point of the optimization problem (7).

\section{B. Optimization over $S_{\mathbf{X}}$ for a Fixed $S_{\mathbf{Q}}$}

Although the optimization problem (7) is not concave in $S_{\mathbf{X}}$, we observe that the Lagrangian (11) is a concave function of $S_{\mathbf{X}}$ for fixed $\bar{S}_{\mathbf{Q}}$, if $\mu \in(0,1]$. Therefore, the maximization

$$
\max _{S \mathbf{X} \succeq \mathbf{0}, \operatorname{tr}\left(S_{\mathbf{X}}\right) \leq P} \mathcal{L}\left(S_{\mathbf{X}}, \bar{S}_{\mathbf{Q}}, \mu\right)
$$

can be solved using standard tools from convex optimization. To verify concavity, note that the Lagrangian (11) can be written as a function of $S_{\mathbf{X}}$ (for a fixed $\bar{S}_{\mathbf{Q}}$ ) as

$$
\begin{aligned}
\mathcal{L}( & \left.S_{\mathbf{X}}, \bar{S}_{\mathbf{Q}}, \mu\right) \\
= & f_{o}\left(S_{\mathbf{X}}, \bar{S}_{\mathbf{Q}}\right)-\mu f_{c}\left(S_{\mathbf{X}}, \bar{S}_{\mathbf{Q}}\right)+\text { const. } \\
= & (1-\mu) \log \left|H S_{\mathbf{X}} H^{\dagger}+S_{\mathbf{i n t}}+\sigma^{2} \mathbf{I}_{(r+d)}+\left[\begin{array}{cc}
\bar{S}_{\mathbf{Q}} & \mathbf{0}_{r \times d} \\
\mathbf{0}_{d \times r} & \mathbf{0}_{d \times d}
\end{array}\right]\right| \\
& +\mu \log \left|H_{s d} S_{\mathbf{X}} H_{s d}^{\dagger}+S_{\text {int }}^{(2,2)}+\sigma^{2} \mathbf{I}_{d}\right|+\text { const. }
\end{aligned}
$$

For $\mu \in(0,1]$, this is a logdet optimization which is concave and can be solved using a numerical package such as CVX [10]. 
The above functional form of the Lagrangian provides intuition about the optimal choice of $S_{\mathbf{X}}$. The Lagrangian is a convex combination of two terms. The first term corresponds to the channel from $\mathbf{X}$ to the combined relay and destination receiver $\left(\widehat{\mathbf{Y}}_{r}, \mathbf{Y}_{d}\right)$, while the second term corresponds to the channel from $\mathbf{X}$ to the destination $\mathbf{Y}_{d}$ alone. For larger values of $C_{0}$ (or equivalently small value of $\mu$ ), the optimal $S_{\mathbf{X}}$ should be close to the water-filling covariance matrix against the combined vector channel $H$. For small values of $C_{0}$, the optimal $S_{\mathbf{X}}$ should be close to the water-filling covariance matrix against the sourcedestination channel $H_{s d}$ alone. The optimal $S_{\mathbf{X}}$ can be thought of as providing a tradeoff between the two.

\section{Optimization of $S_{\mathrm{Q}}$ for a Fixed $S_{\mathbf{X}}$}

We now provide a closed-form solution for the $S_{\mathrm{Q}}$ that maximizes the Lagrangian (12) for a given $\bar{S}_{\mathbf{X}}$, i.e.,

$$
\max _{S_{\mathbf{Q}} \succeq \mathbf{0}} \mathcal{L}\left(\bar{S}_{\mathbf{X}}, S_{\mathbf{Q}}, \mu\right)
$$

The key technique that makes a closed-form solution possible is simultaneous diagonalization by $*$ congruence [3].

For the optimization over $S_{\mathbf{Q}}$ when $\bar{S}_{\mathbf{X}}$ is kept fixed, the objective and constraint functions (8)-(9) can be rewritten as

$$
f_{o}=\log \left|S_{\mathbf{Y}_{r} \mid \mathbf{Y}_{d}}+S_{\mathbf{Q}}\right|-\log \left|S_{\mathbf{Y}_{r} \mid \mathbf{Y}_{d}, \mathbf{X}}+S_{\mathbf{Q}}\right|+\text { const.; }
$$

$$
f_{c}=\log \left|S_{\mathbf{Y}_{r} \mid \mathbf{Y}_{d}}+S_{\mathbf{Q}}\right|-\log \left|S_{\mathbf{Q}}\right|+\text { const.; }
$$

and Lagrangian (15) can be written as:

$$
\begin{aligned}
\mathcal{L}\left(\bar{S}_{\mathbf{X}}, S_{\mathbf{Q}}, \mu\right)= & (1-\mu) \log \left|S_{\mathbf{Y}_{r} \mid \mathbf{Y}_{d}}+S_{\mathbf{Q}}\right|+\mu \log \left|S_{\mathbf{Q}}\right| \\
& -\log \left|S_{\mathbf{Y}_{r} \mid \mathbf{Y}_{d}, \mathbf{X}}+S_{\mathbf{Q}}\right|+\text { const. }
\end{aligned}
$$

where the conditional covariances $S_{\mathbf{Y}_{r} \mid \mathbf{Y}_{d}}$ and $S_{\mathbf{Y}_{r} \mid \mathbf{Y}_{d}, \mathbf{X}}$ are obtained using Schur's complement formula

$$
\begin{aligned}
& S_{\mathbf{Y}_{r} \mid \mathbf{Y}_{d}}=H_{s r} S_{\mathbf{X}} H_{s r}^{\dagger}+S_{\mathbf{i n t}}^{(1,1)}+\sigma^{2} \mathbf{I}_{r}-\left(H_{s r} S_{\mathbf{X}} H_{s d}^{\dagger}+S_{\text {int }}^{(1,2)}\right) \\
& \left(H_{s d} S_{\mathbf{X}} H_{s d}^{\dagger}+S_{\text {int }}^{(2,2)}+\sigma^{2} \mathbf{I}_{d}\right)^{-1}\left(H_{s d} S_{\mathbf{X}} H_{s r}^{\dagger}+S_{\mathbf{i n t}}^{(2,1)}\right),
\end{aligned}
$$

and

$$
\begin{aligned}
& S_{\mathbf{Y}_{r} \mid \mathbf{Y}_{d}, \mathbf{X}}= \\
& H_{s r} S_{\mathbf{X}} H_{s r}^{\dagger}+S_{\text {int }}^{(1,1)}+\sigma^{2} \mathbf{I}_{r}-\left[H_{s r} S_{\mathbf{X}} H_{s d}^{\dagger}+S_{\text {int }}^{(1,2)} H_{s r} S_{\mathbf{X}}\right] \\
& {\left[\begin{array}{cc}
H_{s d} S_{\mathbf{X}} H_{s d}^{\dagger}+S_{\mathbf{i n t}}^{(2,2)}+\sigma^{2} \mathbf{I}_{d} & H_{s d} S_{\mathbf{X}} \\
S_{\mathbf{X}} H_{s d}^{\dagger} & S_{\mathbf{X}}
\end{array}\right]^{-1}\left[\begin{array}{c}
H_{s d} S_{\mathbf{X}} H_{s r}^{\dagger}+S_{\mathbf{i n t}}^{(2,1)} \\
S_{\mathbf{X}} H_{s r}^{\dagger}
\end{array}\right]}
\end{aligned}
$$

where $(.)^{-1}$ denotes the Moore-Penrose pseudoinverse. Our goal is to maximize (18) over $S_{\mathrm{Q}}$. Note that the constraint $S_{\mathrm{Q}} \succeq 0$ is superfluous, since it is already implicit in the domain; (if $\left|S_{\mathbf{Q}}\right|=0$, then $\left.C_{0}=+\infty\right)$. The main step in maximizing (18) is the following simultaneous diagonalization of $S_{\mathbf{Y}_{r} \mid \mathbf{Y}_{d}, \mathbf{X}}$ and $S_{\mathbf{Y}_{r} \mid \mathbf{Y}_{d}}$ based on [3, Corollary 7.6.5]:

Lemma 2 There exists a non-singular matrix $C \in \mathbb{C}^{r \times r}$ such that $C^{\dagger} S_{\mathbf{Y}_{r} \mid \mathbf{Y}_{d}, \mathbf{X}} C=\mathbf{I}_{r}$ and $C^{\dagger} S_{\mathbf{Y}_{r} \mid \mathbf{Y}_{d}} C=\Lambda$, where $\Lambda$ is a diagonal matrix. The diagonal elements $\lambda_{i}$ are called the generalized eigenvalues. Moreover, $\lambda_{i} \geq 1$ for $i=1, \cdots, r$.
Proof: Both $S_{\mathbf{Y}_{r} \mid \mathbf{Y}_{d}}$ and $S_{\mathbf{Y}_{r} \mid \mathbf{Y}_{d}, \mathbf{X}}$ are positive definite matrices. Let $S_{\mathbf{Y}_{r} \mid \mathbf{Y}_{d}, \mathbf{X}}^{-1}=R^{\dagger} R$ be a Cholesky decomposition. Now, consider the eigendecomposition $R S_{\mathbf{Y}_{r} \mid \mathbf{Y}_{d}} R^{\dagger}=$ $V \Lambda V^{\dagger}$. Then $C=R^{\dagger} V$ satisfies $C^{\dagger} S_{\mathbf{Y}_{r} \mid \mathbf{Y}_{d}, \mathbf{X}} C=\mathbf{I}_{r}$ and $C^{\dagger} S_{\mathbf{Y}_{r} \mid \mathbf{Y}_{d}} C=\Lambda$ simultaneously. Moreover, since $C$ is nonsingular, $S_{\mathbf{Y}_{r} \mid \mathbf{Y}_{d}} \succeq S_{\mathbf{Y}_{r} \mid \mathbf{Y}_{d}, \mathbf{X}}$ implies $\Lambda \succeq \mathbf{I}_{r}$.

We note that a special case of this optimization problem has already been considered in [8], where the noises at $\mathbf{Y}_{r}$ and $\mathbf{Y}_{d}$ are independent and $S_{\mathbf{Y}_{r} \mid \mathbf{Y}_{d}, \mathbf{X}}$ is an identity matrix. For the more general correlated noise case, the above simultaneous diagonalization is needed to reduce the matrix optimization problem to scalar optimization.

We also noted that in [9] an equivalent diagonalization approach is taken to solve the same problem, but from a source coding perspective. Instead of diagonalizing $S_{\mathbf{Y}_{r} \mid \mathbf{Y}_{d}, \mathbf{X}}$ and $S_{\mathbf{Y}_{r} \mid \mathbf{Y}_{d}}$, the approach of [9] diagonalizes $S_{\mathbf{X} \mid \mathbf{Y}_{d}}$ and $S_{\mathbf{Y}_{r} \mid \mathbf{Y}_{d}}$ using a singular value decomposition of the matrix $S_{\mathbf{X} \mid \mathbf{Y}_{d}}^{-1 / 2} K_{\mathbf{X} \mathbf{Y}_{r}} S_{\mathbf{Y}_{r} \mid \mathbf{Y}_{d}}^{1 / 2}$, where $K_{\mathbf{X Y}}$ is a certain matrix of regression coefficints. It can be shown that the resulting diagonalization makes $S_{\mathbf{X} \mathbf{Y}_{r} \mid \mathbf{Y}_{d}}^{(1,2)}$ diagonal as well. Subsequently, the diagonal elements from the diagonalization of $S_{\mathbf{X} \mathbf{Y}_{r} \mid \mathbf{Y}_{d}}^{(1,2)}$ are used to find the optimal solution to the overall problem. We observe that the derivations in [9] can be interpreted as an indirect simultaneous diagonalization of $S_{\mathbf{Y}_{r} \mid \mathbf{Y}_{d}, \mathbf{X}}$ and $S_{\mathbf{Y}_{r} \mid \mathbf{Y}_{d}}$, and therefore is equivalent to the approach presented here. However, the direct simultaneous diagonalization of $S_{\mathbf{Y}_{r} \mid \mathbf{Y}_{d}, \mathbf{X}}$ and $S_{\mathbf{Y}_{r} \mid \mathbf{Y}_{d}}$ is simpler and gives more structual insight about the optimal MIMO compress-and-forward strategy.

Returning to the maximization of (18), we now use the approach of [6], [8] to reduce the MIMO optimization problem to the scalar case and to solve the subsequent scalar quantization noise optimization problem. For $\mu \in(0,1]$, the Lagrangian (18) can be written as:

$$
\begin{aligned}
\mathcal{L} & \stackrel{(a)}{=}(1-\mu) \log \left|\Lambda \hat{S}_{\mathbf{Q}}^{-1}+\mathbf{I}_{r}\right|-\log \left|\hat{S}_{\mathbf{Q}}^{-1}+\mathbf{I}_{r}\right|+\text { const. } \\
& \stackrel{(b)}{\leq}(1-\mu) \log \left|\Lambda \Sigma_{\mathbf{Q}}^{-1}+\mathbf{I}_{r}\right|-\log \left|\Sigma_{\mathbf{Q}}^{-1}+\mathbf{I}_{r}\right|+\text { const. }
\end{aligned}
$$

where $(a)$ follows from the change of variable $\hat{S}_{\mathbf{Q}}=C^{\dagger} S_{\mathrm{Q}} C$, with $C$ as in Lemma 2, and (b) follows from [8, Lemma 5] where $\Sigma_{\mathbf{Q}}$ comes from the eigen-decomposition $\hat{S}_{\mathbf{Q}}=U \Sigma_{\mathbf{Q}} U^{\dagger}$. Observe that the equality in $(b)$ is obtained with $U=\mathbf{I}_{r}$. Thus, it is without loss of optimality to restrict $\hat{S}_{\mathrm{Q}}$ to be diagonal.

Let $\Sigma_{\mathbf{Q}}^{i i}$ be the diagonal entries of $\Sigma_{\mathbf{Q}}$. Consider the following change of variable, introduced in [6]:

$$
c_{i}=\log \left(1+\frac{\lambda_{i}}{\Sigma_{\mathbf{Q}}^{i i}}\right), i=1, \ldots, r .
$$

An interpretation of $c_{i}$ is that it is the portion of the available $C_{0}$ assigned for compression of the $i^{t h}$ element of $C \mathbf{Y}_{r}$. Note that we have $\Sigma_{\mathbf{Q}}^{i i} \geq 0$ and $c_{i} \geq 0$. Using (20), the Lagrangian can be written as:

$$
\mathcal{L}=\sum_{i=1}^{r}\left((1-\mu) c_{i}-\log \left(2^{c_{i}}+\lambda_{i}-1\right)\right)+\text { const. }
$$


It can be readily checked that (21) is concave in $c_{i}$ when $\lambda_{i} \geq 1$. The optimal $c_{i}$ is given by

$$
c_{i}^{*}=\left[\log \frac{(1-\mu)\left(\lambda_{i}-1\right)}{\mu}\right]^{+} .
$$

The optimal $\Sigma_{\mathbf{Q}}^{i i, *}$ is given by

$$
\Sigma_{\mathbf{Q}}^{i i, *}= \begin{cases}\frac{\mu}{1-\frac{1}{\lambda_{i}}-\mu} & \mu<1-\frac{1}{\lambda_{i}} \\ +\infty & \mu \geq 1-\frac{1}{\lambda_{i}}\end{cases}
$$

and the optimal $S_{\mathbf{Q}}$ is given by $S_{\mathbf{Q}}^{*}=C^{-\dagger} \Sigma_{\mathbf{Q}}^{*} C^{-1}$.

\section{Simulation Results}

This section demonstrates the effectiveness of using deviceto-device link for enhancing cell-edge throughput in a downlink wireless cellular environment. Consider a picocell deployment with a pico-base-station transmitting at a maximum power of 1 Watt over $10 \mathrm{MHz}$ to a user distance $100 \mathrm{~m}$ away. A second user located nearby acts as a relay as shown in Fig. 1. The background noise power spectral density is assumed to be $-170 \mathrm{dBm} / \mathrm{Hz}$. A channel model with pathloss exponent of 3.76 and $8 \mathrm{~dB}$ shadowing is used. We consider various antenna deployment configurations to demonstrate the effect of antenna pooling for both enhancing the direct signal and for interference mitigation, and to demonstrate the need for using jointly optimized transmit and quantization noise covariance matrices.

As reference, the cut-set upper bound to the capacity of the MIMO relay channel [1, Theorem 4] is stated below:

$$
\mathcal{C} \leq \max _{p(\mathbf{x}), \mathbb{E}\left\{\mathbf{X}^{\dagger} \mathbf{X}\right\} \leq P} \min \left\{I\left(\mathbf{X} ; \mathbf{Y}_{r}, \mathbf{Y}_{d}\right), I\left(\mathbf{X} ; \mathbf{Y}_{d}\right)+C_{0}\right\}
$$

The evaluation of the cut-set bound involves solving an optimization problem. For the Gaussian MIMO relay channel, the optimal input distribution in the maximization problem (24) is multivariate Gaussian. The optimization over the input covariance matrix is a convex optimization problem, which can be solved using standard packages such as CVX [10].

We begin by demonstrating the effect of antenna pooling for enhancing the direct communication between the base-station and the user. Fig. 2 shows the improvement in overall transmission rate using a relay link for a scenario where the transmitting base-station has three antennas $(s=3)$, the relay and destination are each equipped with two antennas $(r=2$ and $d=2)$, and with no intercell interference $(t=0)$. In this scenario, the benefit of adding extra antennas to the destination is in enlarging the signal dimension, as the overall transmission degree-offreedom is limited by the number of antennas at the destination. Thus, it is expected that pooling antennas from the relay is able to improve the overall throughput considerably. As seen in Fig. 2, at $C_{0}=100 \mathrm{Mbps}$, the improvement in throughput by the optimized compress-and-forward relaying scheme is around $92 \mathrm{Mbps}$, achieving an almost 1:1 improvement in the overall rate for each relaying bit. The maximum possible improvement is around $145 \mathrm{Mbps}$, which is achieved with $C_{0}=250 \mathrm{Mbps}$. For smaller values of $C_{0}$, the achievable rate of the optimized compress-and-forward scheme almost meets the cut-set bound.

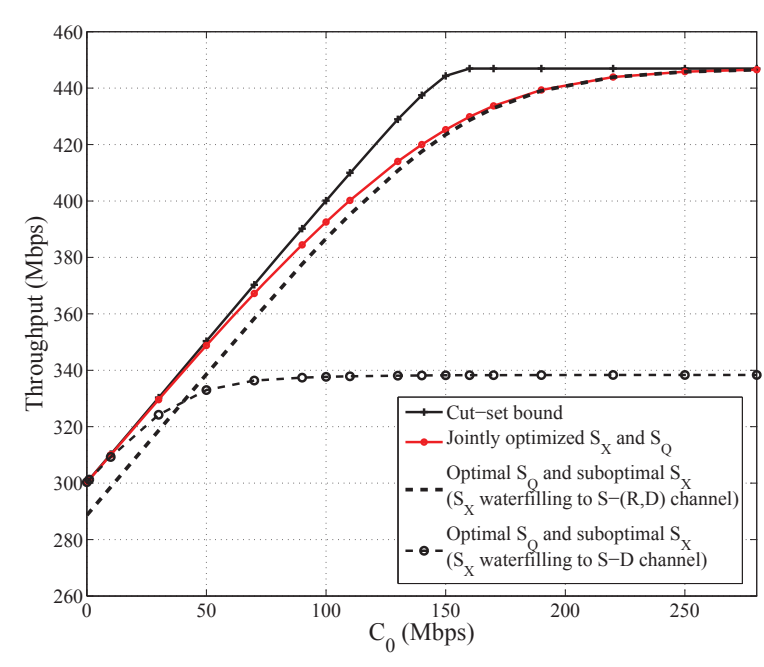

Fig. 2. Throughput improvement due to a device-to-device link of capacity $C_{0}$ for the case where the base-station is equipped with three antennas $(s=3)$, and the relay and destination are equipped with two antennas each $(r=2$ and $d=2$ ) with no inter-cell interference $(t=0)$.

Fig. 2 also demonstrates the need to optimize the input covariance matrix. As suboptimal choices for input covariance, we also plot the achievable rates for the cases where the transmit covariance is, respectively, set to be the water-filling covariance of the source-to-destination and source-to-relay-and-destination point-to-point channels, without considering the effect of finite relay link capacity. For these fixed input covariance matrices, we optimize the quantization noise covariance. Depending on the value of $C_{0}$, each of these covariances can be strictly suboptimal. For small values of $C_{0}$, setting the input covariance to optimize for the source-to-destination channel is close to optimal. However, as $C_{0}$ increases, this $S_{\mathbf{X}}$ fails to achieve the optimal performance. For large values of $C_{0}$, setting the input to optimize for the source-to-relay-and-destination channel is close to optimal, but a gap exits when $C_{0}$ is low.

Fig. 3 presents the results for a similar setup, except that the transmitting base-station now has two antennas, and the relay and destination are equipped with three antennas each, again with no interference (i.e., $s=2, r=3, d=3$, and $t=0$ ). In this scenario the overall degree-of-freedom of the direct link is constrained by the number of baes-station antennas instead of number of antennas at the destination, and relaying does not increase the overall throughput notably. In this case, with $C_{0}=$ $100 \mathrm{Mbps}$, the throughput improvement is only around $17 \mathrm{Mbps}$.

Fig. 4 considers the same setup as Fig. 3, except that now four additional interfering single-antenna pico-base-stations are added as shown in Fig. 1 (i.e., $s=2, r=3, d=3$, and $t=4$ ). The interfering base-stations are placed on a hexagonal grid $200 \mathrm{~m}$ away from the center base-station. Due to interference, the throughput without the relay (i.e., with $C_{0}=0$ ) is now considerably lower, but the optimized use of the relay link is able to improve the throughput significantly. This is because, due to the common intercell interference, the noises at the relay and destination are highly correlated. By exploiting such noise 


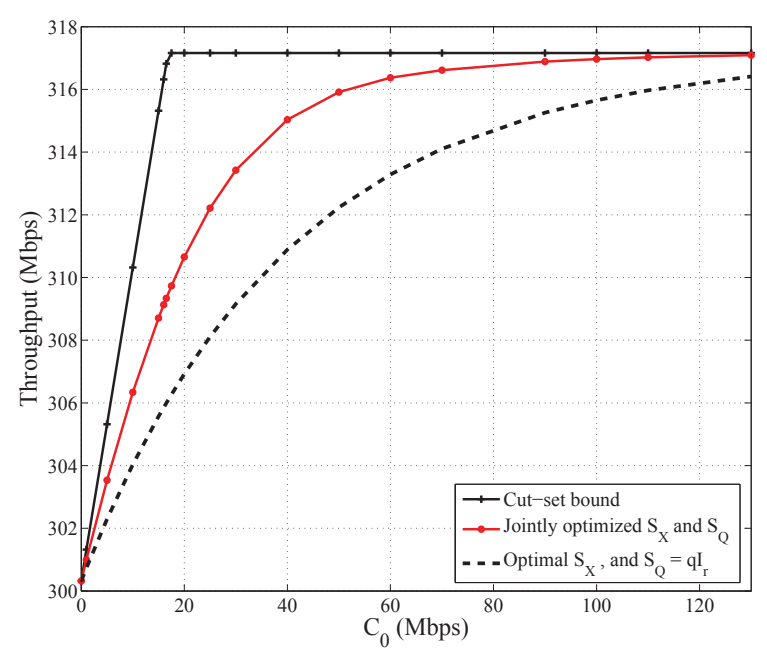

Fig. 3. Throughput improvement due to a device-to-device link of capacity $C_{0}$ for the case where the base-station is equipped with two antennas $(s=2)$, and the relay and destination are each equipped with three antennas $(r=3$ and $d=3)$ with no inter-cell interference $(t=0)$.

correlation using the relay link, the destination can effectively pool the three relay antennas together with the three existing antennas of its own to create a $2 \times 6$ overall MIMO channel (i.e., $s=2, r+d=6$ ). This enables the rank-four interference (i.e., $t=4$ ) to be rejected completely, creating an effective $2 \times 2$ interference-free overall channel. As seen in Fig. 4, at around $C_{0}=100 \mathrm{Mbps}$, the improvement in throughput brought by the optimized use of the compress-and-forward relay link is around $97 \mathrm{Mbps}$. At small $C_{0}$, relaying again achieves almost 1:1 improvement in the direct transmission rate for each relaying bit. It is worth noting that the overall throughput at large $C_{0}$ in Fig. 4 is close to the achievable rate of the $C_{0}=0$ scenario in Figs. 2 and 3, illustrating the almost complete interference rejection capability of optimized compress-and-forward relaying.

Figs. 3 and 4 also demonstrate the importance of optimizing the quantization noise covariance matrix. For comparison purpose, the achievable rate for a simple suboptimal $S_{\mathrm{Q}}$ is also plotted, where $S_{\mathbf{Q}}=q \mathbf{I}_{r}$ and $q$ is set to satisfy the relay rate constraint with equality, for the optimal $S_{\mathbf{X}}$ obtained from Algorithm 1. This simple choice of $S_{\mathbf{Q}}$ results in a strictly suboptimal performance as shown in Figs. 3 and 4.

\section{CONCLUSION}

This paper demonstrates that the optimized use of compressand-forward in cooperative device-to-device communication can significantly improve the throughput of cell-edge users in a wireless cellular network. The relay link allows two users to effectively pool their antennas together. It can benefit the overall transmission rate in two ways. First, antenna pooling enlarges the receiver dimensions thus allowing additional transmission degree-of-freedom from the base-station. Second and more importantly, antenna pooling enables joint interference rejection across the user terminals, thus allowing more interference-free dimensions for direct transmission.

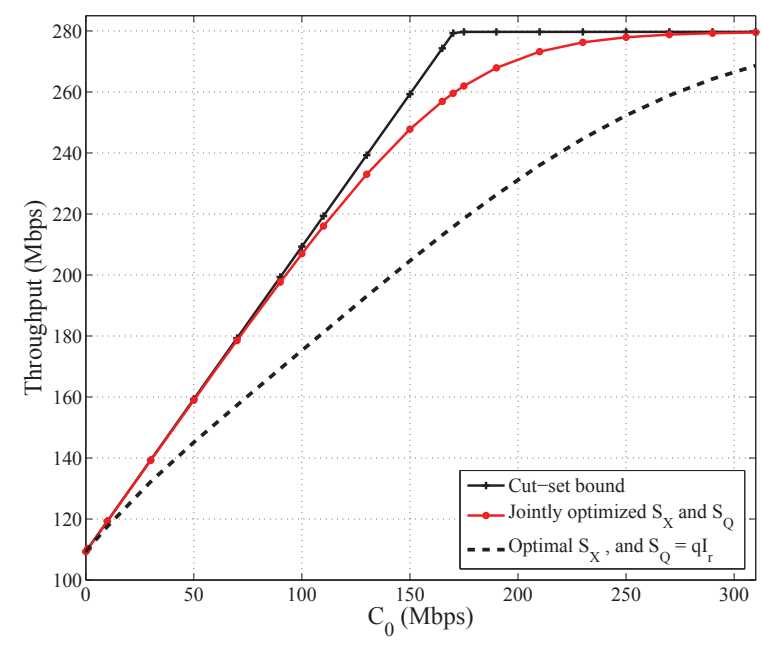

Fig. 4. Throughput improvement due to a device-to-device link of capacity $C_{0}$ for the case where the base-station is equipped with two antennas $(s=2)$, and the relay and destination are each equipped with three antennas ( $r=3$ and $d=3$ ) with four inter-cell interference sources $(t=4)$.

This paper proposes an alternating optimization approach for the optimization of the transmit and quantization covariance matrices in compress-and-forward relaying. The optimization of quantization covariance involves a simultaneous diagonalization by *congruence of two conditional covariance matrices and optimal allocation of quantization rates. The optimization of transmit covariance involves solving a convex logdet problem. The iterative optimization algorithm converges to the stationary point of the Lagrangian, which leads to a KKT solution of the overall throughput maximization problem.

\section{REFERENCES}

[1] T. Cover and A. El Gamal, "Capacity theorems for the relay channel," IEEE Trans. Inf. Theory, vol. 25, no. 5, pp. 572-584, Sep. 1979.

[2] G. Kramer, M. Gastpar, and P. Gupta, "Cooperative strategies and capacity theorems for relay networks," IEEE Trans. Inf. Theory, vol. 51, no. 9, pp. 3037 - 3063, Sept. 2005.

[3] R. A. Horn and C. R. Johnson, Matrix Analysis. Cambridge University Press, 1990

[4] L. Zhang, J. Jiang, A. Goldsmith, and S. Cui, "Study of Gaussian relay channels with correlated noises," IEEE Trans. Commun., vol. 59, no. 3 , pp. 863-876, Mar. 2011.

[5] L. Zhou and W. Yu, "Capacity of the Gaussian relay channel with correlated noises to within a constant gap," IEEE Commun. Lett., vol. 16, no. 1, pp. 2-5, Nov. 2012.

[6] S. Simoens, O. Muoz-Medina, J. Vidal, and A. del Coso, "Compress-andforward cooperative MIMO relaying with full channel state information," IEEE Trans. Signal Process., vol. 58, no. 2, pp. 781-791, Feb. 2010.

[7] D. P. Bertsekas, Nonlinear Programing. Athena Scientific, 2003.

[8] A. del Coso and S. Simoens, "Distributed compression for MIMO coordinated networks with a backhaul constraint," IEEE Trans. Wireless Commun., vol. 8, no. 9, pp. 4698-4709, Sep. 2009.

[9] C. Tian and J. Chen, "Remote vector gaussian source coding with decoder side information under mutual information and distortion constraints," IEEE Trans. Inf. Theory, vol. 55, no. 10, pp. 4676-4680, Oct. 2009.

[10] CVX Research, Inc., "CVX: Matlab software for disciplined convex programming, version 2.0 beta," Sep. 2012. [Online]. Available: http://cvxr.com/cvx 